\title{
The Transcendentist Theory of Persistence
}

\author{
[penultimate draft; please cite only the published version]
}

This paper develops a new endurantistic theory of persistence. The theory is built around one basic tenet, which concerns the relation between objects and time, i.e. existence at a time. According to this tenet, for an object to exist at a time is for it to participate in events that are located at that time. For reasons that will become obvious later, I call this tenet transcendentism.

I will argue that transcendentism reveals itself to be a semantically grounded and metaphysically fruitful choice. Semantically grounded, insofar as a semantic analysis of our ordinary temporal talk seem to strongly favour it over rivals. Metaphysically fruitful, insofar as the theory of persistence that can be built around it - the transcendentist theory of persistence, to give it a name - requires neither temporal parts nor the metaphysically problematic assumptions to which all extant versions of endurantism are committed, such as the possibility of extended simples or of multilocation.

In $\S 1$ I present the current state of the debate between endurantism and perdurantism, with a focus on what I call the locative turn. Thanks to the

locative turn, it is now possible to distinguish at least two different forms of endurantism. However, both are based on problematic assumptions. In $\S 2, \mathrm{I}$ sketch transcendentism and the theory of persistence that can be built around it, as well as their semantic groundedness and metaphysical fruitfulness. 


\section{Endurantism, perdurantism and the locative turn}

Let us say that an object persists through time if and only if it exists at various times. Nowadays most philosophers agree that objects persist through time ${ }^{1}$. Yet, they disagree on how they do so. The bulk of their disagreement is whether or not such objects have temporal parts ${ }^{2}$. So-called perdurantists think they do, so-called endurantists think they do not. The conflict between endurantists and perdurantists is made up of several well-known arguments against endurantism. Perdurantists think that temporal parts are needed in order to account for the phenomena such as change, mereological coincidence and vagueness. From their side, endurantists insist that they can account for those phenomena just as well without temporal parts, and so find the whole idea that objects have temporal parts unmotived, let alone per se implausible.

Yet another challenge for the endurantist concerns the very definition of his view. Perdurantism may be based on a prima facie implausible tenet, yet it is has a clear and positive definition: according to it, persisting objects have temporal parts. On the other hand, endurantism is often characterized in purely negative terms, as the thesis that objects do not have temporal parts. Sometimes, endurantism is defined in positive terms, as the thesis that objects are wholly present at various times. Still, this definition rests on the notion of being "wholly present", which many take to be unclear. What does it mean that $x$ is wholly present at a time $t$ ? It does not mean that every part that $x$ has at any

\footnotetext{
${ }^{1}$ With the possible exception of stage theorists such as (Hawley, 2001; Sider, 2001; Varzi, 2003).

${ }^{2}$ Let $x$ be a temporal part of $y$ just in case $x$ is a part of $y$, exists only at a time $t$, and overlaps everything that is part of $y$ and exists at $t$. Another way of putting that is that temporal parts are what you get when you slice an entity along the temporal dimension, so that you have different parts of it at different times. Events are usually taken to have these kinds of parts, so that, e.g. a football match divides into two temporal parts, the first and the second half.
} 
time is part of $x$ at $t$, otherwise it would be impossible for $x$ to change parts over time. Neither does that mean that every part that $x$ has at $t$ is a part of $x$ at $t$, because that is a trivial claim that also the perdurantist would like to hold. Perhaps, it just means that $x$ has no temporal part at $t$. But in this case, to be wholly present at various times would just mean to persist without having temporal parts, so that endurantism would again turn out to be defined in purely negative terms.

Should an endurantist give up to this challenge, and admit that endurantism is intuitive but yet unclear? Thankfully for the endurantist, recent work on the notion of location can help him in doing much better. Let me first say a few words on the notion of location, and then explain how it can help the endurantist in defining his view.

Let exact location be the relation between an entity $x$ and a region $r$ of a dimension $d$ which holds just in case $x$ and $r$ have the same shape, boundaries, size, and stand in the same distance relations in $d$ with other entities (Gilmore, $2006,2007,2008)$. For example, the exact spatial location of an ice cube is that cubic spatial region where the cube perfectly fits and is as distant to everything else as the cube is. Exact location has to be contrasted to other notions of location, most notably weak location. A weak location of an entity is a region that is not completely free of that entity. In mereological terms, we can define a weak location of $x$ as any region that overlaps the exact location of $x$. The ice cube, for example, is weakly located at the region of my glass, of the room, of the universe as well as at any sub-region of its exact location.

The term location comes from the spatial case, but there seems to be no reason to deny on principle the possibility that a perfectly similar relation concerns other dimensions as well. Quite the contrary, metaphysicians feel free to 
Figure 1.1: Weak and exact location: the round entity $x$ is weakly located at regions $r 1, r 2, r 3, r 5$, is exactly located at $r 1$, and is neither exactly nor weakly located at $r 4$.

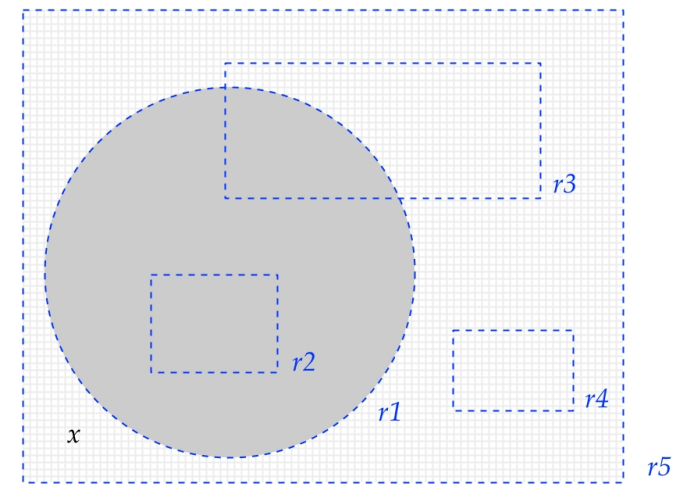

speak of temporal and spatiotemporal location as well (Parsons, 2007; Gilmore, 2006, 2007, 2008; Sattig, 2006; Balashov, 2010; Bittner and Donnelly, 2006). If the relevant dimension may change, the conditions for being exactly located do not. For example, take the temporal case. An entity $x$ is exactly located at a temporal region $t$, be it an instant or an interval of time, just in case, $x$ and $t$ share shape, boundaries, size, and distance relations. For example, the exact temporal location of World War II is a six-years interval of time spanning from 1939 to 1945 . WWII shares with that region its shape and size - both are temporally extended and last six years - and shares with it temporal distance relations - both are, for example, 21 years after WWI.

Let us now focus on temporal location. Nowadays most philosophers agree that objects are located in time ${ }^{3}$. Yet, they disagree at which regions of time they are exactly located. Some of them believe that objects have one exact temporal location. They take this unique exact temporal location to be the interval of an object's persistence - the one which has as boundaries the instants at which the persisting object comes into being and goes out of existence, respectively. Call these philosophers unilocists. Some others believe that objects have

\footnotetext{
${ }^{3}$ Pace Simons (2014) andFine (2006).
} 
several exact temporal locations. They think that a persisting object is exactly located at all and only the instants included in the interval of their persistence. Call these philosophers multilocists.

Let us explore a bit these two options. First, we can lay down a straightforward connection between unilocationism and an idea that has long been connected to, or identified with, perdurantism, i.e. the idea that objects are extended both in space and in time. Let us recall that exact location is linked to size: if $x$ is exactly located at $r, x$ and $r$ have the same size. In the temporal case, size boils down to temporal extension: something that is located in time can either be temporally extended - iff it is exactly located at an interval - or unextended - if it is exactly located at instants only -. Hence, multi-locists have it that objects are not extended in time while uni-locists have it that objects are extended in time. Second, another straightforward path lead us from the idea that objects are extended in space and time to another idea that has long been connected to, or identified with, perdurantism, i.e. the idea that objects have a four-dimensional shape. If we replace space and time with spacetime, the claim that objects are extended in space and time translates into the claim that objects are exactly located at a four-dimensional spatiotemporal region. Recall that exact location is linked to shape: if $x$ is exactly located at $r, x$ and $r$ have the same shape. Given that uni-locists have it that objects are exactly located at four-dimensional regions of spacetime, they also have it that objects themselves have a four-dimensional shape. Let us now have a look at the multilocationist picture. Given that muli-locationism has it that objects are exactly located at instants of time, and given that instants are unextended, it follows that according to the multi-locationist objects are temporally unextended. And once we replace space and time with spacetime, the multi-locist ends up defending the view that objects have a three-dimensional shape, given that their 
exact spatiotemporal location can at most be a three-dimensional, temporally unextended, region. Both claims, i.e. that objects are not extended in time and that objects are three-dimensional, have traditionally been connected to, or identified with, endurantism.

Figure 1.2: Multilocationism and unilocationism: a three-dimensional object occupies a four-dimensional region by being multi-located through it, while a four-dimensional object occupies that region by being exactly located at it.
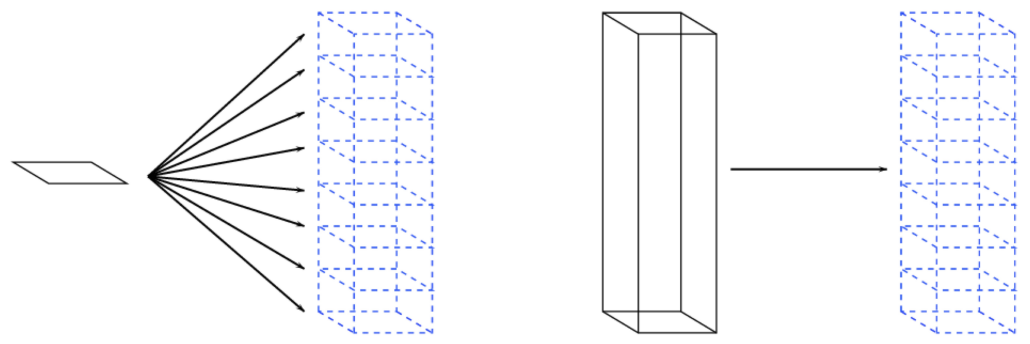

Unilocationism implies claims that are traditionally identified with perdurantism, while multilocationism implies claims that are traditionally identified with endurantism. At this point one may be tempted to say that unilocationism simply is perdurantism in disguise and that the same holds for multilocationism and endurantism. However, notice that the two disputes differ in content. The endurantism/perdurantism dispute concerns the having or not of temporal parts; the uni-/multilocationism dispute concerns the exact temporal location and geometry of an entity. Moreover, it has been suggested that the two disputes do not align with one another, for unilocationism - as a locational thesis may perhaps be compatible with endurantism - as a thesis concerning temporal parts -.

More interestingly, if we keep the two disputes apart, we are finally able to define in a clear and positive way not only one, but actually two, forms of endurantism. The first and more obvious one simply is multilocationism. 
Recall that according to multi-locism an object persists through time by being exactly temporally located at each instant of its persistence (Gilmore, 2004, 2006; Sattig, 2006; Bittner and Donnelly, 2006). This view qualifies as a form of endurantism, for multilocationism implies endurantism: since what includes all of the object at a time is the object itself exactly located there, it cannot be a (proper) part of it; a fortiori, it is not a (proper) temporal part of it.

The second and less obvious form of endurantism that we are now in a position to define makes appeal to the notion of an extended simple. An extended simple is an entity that is both extended in a dimension - i.e. exactly located at an extended region of that dimension - and mereologically simple, i.e. without any proper parts. If extended simples are possible, an endurantist may endorse the unilocationist view, and so claim that a persisting object is temporally extended, and yet think that this object is an extended simple(Parsons, 2000, 2007). This view qualifies as a form of endurantism, for, once again, it implies it: the persisting objects has no (proper) temporal parts because it has no (proper) parts at all. For obvious reasons, I will label this view simplism.

So far I have characterized simplism and multilocationism by means of their definitions. Still, it is interesting to see how things work at a deeper level, by analyzing the constitutive postulates on which the views depend. On the one hand, multilocationism depends on the possibility of multilocation, i.e. the possibility that an entity be exactly located at more than one region of a dimension. In other words, multilocationism requires the negation of a principle called the functionality of exact location, according to which exact location is functional: an entity can have at most one exact location at a dimension:

[functionality] $x @ r \wedge x @ s \rightarrow r=s$

On the other hand, simplism depends on the possibility of extended simples. In other words, simplism requires the negation of a principle called arbitrary 
partition, a.k.a. the doctrine of arbitrary undetached parts [DAUP], according to which extension implies composition: entities have a proper part for each sub-region of their exact location:

[DAUP] $\quad x @ r \rightarrow \forall s(s \ll r \rightarrow \exists y(y \ll x \wedge y @ s))$

Are the constitutive postulates of these two views any plausible? Let us try to answer this question step by step, and let us being with extended simples.

Are extended simples possible? In some theories of location, extended simples turn out to be conceptually impossible, e.g. in (Casati and Varzi, 1999). And even if there are theories of location that are formally compatible with extended simples, many think that these entities are simply conceptually or metaphysically impossible, because extension implies mereological composition. However, notice that DAUP is not still enough to prove that extended entities have proper parts. In particular, two additional assumptions need to be made. The first one is that the given entity is not multilocated. Otherwise, it could be multilocated at each part of any of its exact locations and hence not violate DAUP. (Curiously enough, this fact shows that - contrary to what many thought (van Inwagen, 1990; Sider, 2001) - endurantism, in its multilocationist form, seems to be fully compatible with the doctrine of arbitrary undetached parts). On the other hand, if an entity has just one exact location the parts located at the proper parts of their exact location must be proper parts. To be sure, suppose $x$ is located at $r$, and there is an $r^{\prime}$ which is a proper part of $r$. If it is a proper part of $r$, it must be numerically distinct from it. Hence, $x$ cannot be exactly located there. Hence, the $y$ that, by DAUP, is exactly located at $r^{\prime}$ is numerically distinct from $x$. Hence, again by DAUP, it is a proper part of it. The second additional assumption that has to be made to prove that if DAUP is true then extended entities have proper parts is that extended regions themselves have proper parts. This sounds like an innocent 
assumption. However, in recent years, some argued in favour of extended simple regions (Braddon-Mitchell and Miller, 2006). If one of the two assumptions is dropped, there might be extended simples even if DAUP is true. However, the first assumption is explicitly denied by Simplism. On the other hand, the second assumption does not serve well Simplism after all. Even if there were extended simple regions, they would be extremely small, or at least existing arguments support only the existence of extremely small extended simple regions (Braddon-Mitchell and Miller, 2006). Hence, the theories would not be able to make sense of common cases of persistence in which substances persist for a reasonably extended amount of time. A theory that allows for the persistence of short-living entities only would not be much useful. Simplism really has to drop DAUP completely, if it wants to make sense of ordinary cases of persistence. And this is unfortunate, because many think that DAUP should be conceptually or metaphysically necessarily true (Braddon-Mitchell and Miller, 2006; Casati and Varzi, 1996, 1999; Hofweber and Velleman, 2011; Sider, 1997).

Let us now turn to the possibility of multilocation. Many take the very idea of multilocation to be incoherent or even non-sense. The incoherence charge comes from a battery of alleged paradoxes that the hypothesis generates. About the paradoxes, much has been written and I limit myself to review them briefly. According to a first alleged paradox, any multilocated entity would constitute a contradiction. In fact, when an entity is multilocated at regions $r$ and $r$, it will be at a zero distance from itself but also at a non-zero - basically the distance $n$ that divides $r$ and $r^{\prime}$ - distance from itself (Ehring, 2002; Gilmore, 2003). Another famous paradox regarding multilocation goes as follows. If an entity persists through an interval of time in the way multilocationism says it does, it will be temporally unextended. However, there is something, the sum of all "instances" of that substance, one for each instant of the interval, which seems 
to be temporally extended. And such a sum simply is the multilocated substance itself, because summing an entity with itself will always return nothing else than the entity itself again. Hence, the entity is both temporally extended and instantaneous (Barker and Dowe, 2003, 2005). Other paradoxes show the incompatibility of multilocation, time travel and widely accepted principles of mereology, such as antisymmetry, transitivity or weak supplementation (Effingham and Robson, 2007; Gilmore, 2007; Kleinschmidt, 2011). The non-sense charge against multilocationism comes from appearence of there being no way of making sense of exact location that does not make multilocation hopeless. All definitions of exact location make multilocation impossible, and taking it as a primitive rules out cases that seem to be conceptually possible (Parsons, 2007).

With multilocationism and simplism in hand, the endurantist has finally laid to rest the challange that endurantism is unclear and undefinable in positive terms. The search for a clear definition for endurantism has led to not only one, but actually two competing forms of endurantism. More generally, the introduction of the concept of location in the persistence debate has radically reshaped the debate itself, so that one is tempted to talk of a veritable turn, a locative turn, here. Yet both new forms of endurantism rest on postulates which have been taken to be conceptually, logically, or metaphysically problematic and counter-intuitive. The outcome of this discussion is somehow surprisingly uncomfortable for the endurantist. Before the locative turn, endurantism seemed to be intuitive but yet unclear. After the locative turn endurantism seems to be clear but counter-intuitive. 


\section{Transcendentism}

In the previous section, I explained how the introduction of the concept of location in the persistence debate - the locative turn - brought the debate to a deeper level of analysis. After this turn, the various theories in play are no longer characterized by their attitude towards temporal parts, but also and primarily by the way in which they say objects exist at extended regions of time, e.g. by being exactly located at, or multilocated through, such extended regions. This turn allowed us to distinguish two forms of endurantism, i.e. multilocationism and simplism. However, this turn also highlighted the fact that both forms of endurantism rely on highly problematic assumptions. This result seems to leave the persistence debate in a sort of impasse, especially from the endurantistic point of view, for endurantists seem to have lost their alleged advantage of holding the most intuitive view.

This section will introduce transcendentism as a solution to this impasse, for transcendentism, as we shall see, is a form of endurantism that is free of the problematic postulates of both multilocationism and simplism. Recall that persistence is defined in terms of existence at a time: for an object to persist, it is said, is for it to exist at different times. The notion of existence at a time will play a central role in this section, so let us begin by focussing on it for a moment.

Existence at a time is the relation linking an object to time, or more precisely, to the instants and intervals of time at which the object is present. So, for example, I exist now and at any instant or interval of the past year, as well as at any instant of the interval of my life and any temporal region overlapping such instants. Existence at a time is different from quantification, or existence simpliciter (Sider, 2001, 58-59). Existence at a time is a relation, while quantification is not. Quantification, unlike existence at a time, concerns all kinds 
of entities, also those that are outside of time, if any. Quantification is what determines what makes part of our ontology, while existence at a time simply informs us about the times at which an object is present ${ }^{4}$.

To my knowledge, not much has been written about existence at a time. One outstanding question concerning existence at a time is: what is it for an object to exist at a time? After the locative turn, extant theories of persistence carry with them an implicit answer to this question. According to this answer, existence at a time is location (weak location, more precisely): for an object to exist at a time is for it to be weakly located at that time. I think that the assumption is false, and that the impasse in which the debate is stuck is basically due to this false assumption. In what follows, I will explain why I think that the assumption is false, and will put forward an alternative view. I will begin with a parallel debate concerning the spatial profile of universals that originally inspired the view and that will help us bring the picture to light.

\subsection{Transcendentism: the analogy with universals}

In the Plurality of Worlds, David Lewis traces a parallelism between endurantism on the one hand and the spatial profile of universals on the other. He writes (Lewis, 1986, 202)

Endurance corresponds to the way a universal would be wholly present wherever and whenever it is instantiated.

The parallelism suggests that in order to better understand endurantism, we should have a look at the way in which universals are in space. And there, there are basically two views, i.e. immanentism and transcendentism. According

\footnotetext{
${ }^{4} \mathrm{~A}$ conflation between existence at a time and existence simpliciter may be due to the closeness of their names. Perhaps, to avoid this ambiguity, one may think to replace existence at a time with presence at a time. Yet, this move would only replace an ambiguity with another ambiguity, the one between presence at a time and the tensional present of presentism, the growing block and the moving spotlight. For this reason, I will stick to standard terminology here.
} 
to immanentism, universals are present in space by being located at regions thereof. According to transcendentism, universals are present in space only by being instantiated by objects which, in turn, are located at regions of space ${ }^{5}$.

Figure 2.1: Immanentism and transcendentism about universals.
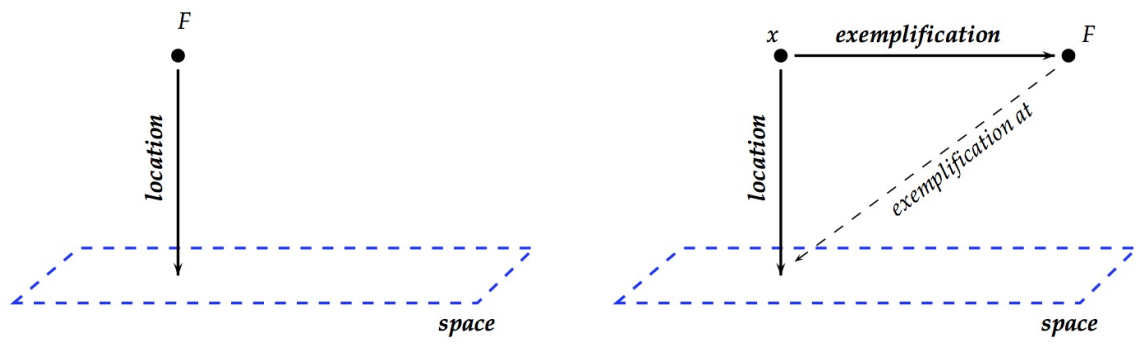

Notice that immanentism claims that universals are present in space by being located at regions thereof, but it does not yet specify in which way universals are located at several regions theoreof. It does not claim, for example, that they are multi-located, because have an exact location for each place where they are instantiated (Gilmore, 2003), or that they are extended, scattered, entities that are singly located at the union of the location of their instances (Effingham, 2015). On the other hand, transcendentism denies both options, because it denies that universals are located in space at all.

One question about transcendentism concerns its precise formulation. Transcendentism says that for a universal to be instantiated at a region of space is for it to be instantiated by an object which is located at that region. The transcendentist claim has the following form: for $x$ to be $F$ is for $x$ to be $G$.

\footnotetext{
${ }^{5}$ To be more precise, we should distinguish between two senses in which a conception of universals is immanentist or transcendentist, viz. a locative sense and an ontological sense. According to the ontological sense, universals are immanent iff they need to be instantiated in order to exist; otherwise they are transcendent (Lowe, 2006). In what follows, I will focus on the locative sense only.
} 
But what does it mean here that for $x$ to be $F$ is for $x$ to be $G$ ? Here are two unsuccessful ways in which the claim can be read. According to the first reading, the transcendentist is proposing a simple material equivalence: a universal is instantiated at a region iff an object instantiates it and is located at that region. According to the second reading, the transcendentist is proposing a grounding ${ }^{6}$ claim: a universal is instantiated at a region in virtue of its being instantiated by an object which is located at that region. I do not think these two readings really make justice to the transcendentist position. To see this, notice that also the immanentist could agree on them. The immanentist believes that redness is located here. She could also agree with the grounding claim and say that redness is located here because redness is instantiated by my cup which is located here. And it is likely that the immanentist will agree on the material equivalence as well: the immanentist would not deny that redness is located wherever there is something that instantiates it, and vice versa.

I think that the transcendentist position is better understood in terms of reduction: a universal's being instantiated at a region reduces to its being instantiated by an object that is located at that region ${ }^{7}$. What distinguishes grounding and reduction? An example coming from the philosophy of mind may be enlightening on this regard. As regards the mental, one may be an eliminativist, and say that there are no mental entities at all (Rorty, 1965; Churchland, 1981, 1984, 1986; Stich, 1983), or one may be a realist, and say that there are mental entities, such as mental episodes, for example. Further, a realist about the mental can be a reductionist or a non-reductionist. According to the reductionist, mental episodes reduce to physical ones, so that anytime you have a mental episode and its physical correlate, you do not have two episodes, but only one

\footnotetext{
${ }^{6}$ Let grounding be what links facts when one of them obtain in virtue of, or because of, some others (Correia, 2005; Fine, 2012; Rosen, 2010; Schaffer, 2012).

${ }^{7}$ More on reduction and its distinction from grounding can be found in (deRosset, 2013; King, 1998; Rosen, 2010).
} 
(Lewis, 1970; Armstrong, 1968; Braddon-Mitchell, 2003; Jackson, 2003). According to the non-reductionist, mental episodes do not reduce to physical ones, and thus when we talk about a mental episode and its physical correlate, we are really speaking about two different entities. Finally, a non-reductionist may still hold that even though the mental does not reduce to, it still is grounded in, the physical, because the physical correlate of a mental episode plays a role in the metaphysical explanation of the occurence of the mental one (Correia, 2005; Correia and Schnieder, 2012; Dasgupta, 2014; Fine, 2012; Schaffer, 2009). From this example, we may draw the following consequences. Reduction is somehow akin to identity: if the mental reduces to the physical, then the mental episode and its physical correlate are just one episode. On the other hand, grounding posits a relation between two facts, which still remain numerically distinct, so that both need to be admitted in our ontology: when one claims that the mental is grounded in the physical one is not thereby committing oneself to the claim that physical and mental states are numerically identical. Defined in reduction terms, transcendentism is incompatible with immanentism, which claims that we should posit in our ontology both a relation of location between universals and space and the two relations linking universals to objects and objects to space. I take this fact to be the core of their disagreement.

\section{$2.2 \quad$ Immanentism and commonsense}

I trust I've said enough about how transcendentism and immanentism should be characterized in the context of universals. Let us now go back to the case of objects and time. There too, we may individuate an immanentist view about how objects are in time - about what it is for the to exist at a time, to use the standard terminology introduced before. According to this immanentist view, for an object to exist at a time is for it to be located at that time. As already 
stated, immanentism is an implicit assumption of all theories of persistence described so far. This implcit assumption that existence at a time is location has proven itself useful insofar as recent work on the concept of location proper have been used to distinguish different theories of persistence and define those already existing. But of course, the usefulness of an assumption does not make that assumption true. Quite the contrary, I think that immanentism about existence at a time is false.

Here is a first way in which someone may argue against immanentism. Recall that for something to be located at a region is for that thing to have the same shape, boundaries and size of that region and to stand with other entities in the same distance relations had by the region (Gilmore, 2006, 2007). If this is the case, for an object to be located in time implies for it to have shapes, sizes, and to enter distance relations with other entities. And this is prima facie implausible. Consider the following sentence that ascribes a temporal distance to two objects:

Aristotle is fifteen years later than Socrates.

This first sentence surely sounds awkward, as well as many others ascribing temporal distance to objects. One may be tempted to resist, and say that when one asserts (1), what one really mean is that

Aristotle's birth is fifteen years later than Socrates' death.

This second sentence sounds more kosher. However, notice that in this second sentence temporal distance relations are not ascribed to objects - Aristotle and Socrates -, but rather to events in which Aristotle and Socrates are somehow involved - their death and birth, respectively. So the claim that sentences ascribing temporal distance relations to objects sound awkward still stand.

What should we do with these semantic considerations against immanentism? It goes without saying that it would be illegitimate to conclude from 
them that immanentism is false. After all, we may build up semantic considerations against heliocentrism, special relativity and quantum mechanics, and hardly anybody would grant those considerations any theoretical force. I believe that immanentism is false, but I think that the best way to show that it is false consists in highlighting its metaphysical problems and how such problems may be solved by means of a theory built on its negation. Still, there is something that may be read out of the semantics of our natural language, or at least something that such semantics may help spelling out, i.e. our deep-rooted metaphysical intuitions. It is controversial whether intuitions should have any weight in philosophy, yet many metaphysicians think they play some role in metaphysics. Those philosophers may be somehow moved by these semantic considerations against immanentism.

Another way in which these semantic considerations may play a role in our debate is suggesting a way of framing a transcendentist theory of existence at a time. Recall that the heart of a transcendentist theory is that it reduces the relation between an entity and a dimension by means of some proxy of that entity in the give dimension. So if there is a transcendentist theory of existence at a time, its aim should be to reduce existence at a time by means of some proxy of objects in time. This role of proxy of objects in time may be played by different kinds of entities, such as states of affairs, tropes or facts. However, the semantic considerations about our temporal talk given before suggest that events may be the most natural candidates for this role.

\subsection{Events}

I will here take events to be anything that happens or occurs. There are simple and short events, such as a collision, a heating process, the uniform movement of a body, or the mental state I am currently in, but also more complex and long 
events, such as a football match, the sum of all mental episodes constituting my stream of consciousness during the last hour, a football match, my life, the history of the universe.

Events have an intimate relation to time. Since events have temporal boundaries, a temporal extension (equal or greater than zero), and enter temporal distance relations, I see no problem in admitting that this relation can be described as a relation of location. Some events persist through time, and when they do, they do so by perduring, i.e. by being exactly located at the interval of their persistence and by divinding into temporal parts throughout $i t^{8}$. For example, a life divides into different phases, such as childhood and adultness. Those phases are parts of a life. They sure are not spatial parts of that life, because they are not distinguished by being at different places. Rather, they are temporal parts of the life, because they are distinguished by occurring at different times. I say that some events perdure because I do not exclude that there may be instantaneous events, such as a collision, or an instantaneous temporal part of an event. Quite the contrary, I think it is plausible that events have a temporal part at each instant of their persistence. Otherwise, they would either need to be temporally multi-located or temporally extended simples.

Another important relation that events have, is the one they have with their subjects, i.e. the objects that participate in them. I call this relation participation. I will then say that I am the participant in my current events, my mental episodes and all events making up my life. Nothing will hang on that later, but I think that participation must be distinguished from parthood, because objects are not parts of events. After all, no matter the way in which you cut an event, what you get will only be smaller events, and not entities of a completely different ontological category. Perhaps, participation may be

\footnotetext{
${ }^{8}$ Or at least standard lore has it that way (Casati and Varzi, 1999; Dretske, 1967; Geach, 1969; Mellor, 1998; Russell, 1903), pace Stout (1997); Galton (2006).
} 
another form of constitution, or at least so it is sometimes assumed to be (Kim, 1976; Lombard, 1986; Bennett, 1988). Another option, if one thinks that events ontologically depend on objects (or the other way round) one may be to define it in terms of ontological dependence or grounding (Correia, 2005; Fine, 2012; Lowe, 1998; Schaffer, 2009; Schnieder, 2011)

Participation may come in a loose and a strict sense. In the strict sense, I am a participant in my life and all parts thereof; and an object is a participant in its history and all parts thereof. In the loose sense, I am a participant in any event in which I play any role, no matter how small it is. The history of the universe is an event in which I am a loose participant, because I play a role in it, but no strict participant, because it is not a part of my life. Of course, we do not need two primitive notions here. For example, we may define loose participation in terms of strict participation: I loosely participate in any event that overlaps an event in which I strictly participate. (As we shall see, strict participation is the one in play in transcendentism.)

\subsection{Transcendentism and the transcendentist theory of persistence}

We are now in a position to properly define transcendentism about the relation between objects and time. According to it, the existence of an object at a time is reduced to its participating in events that are weakly located at that time. For example, for me to exist now is for me to participate in the current temporal part of my life and for my computer to have existed five minutes ago is for it to have participated in the temporal part of its history located at that time. In a motto, transcendentism has it that for an object to be in time is for it to have a history.

Once transcendentism is put forward, its definition will affect other notions 
Figure 2.2: Immanentism and transcendentism about existence at a time.

Immanentism

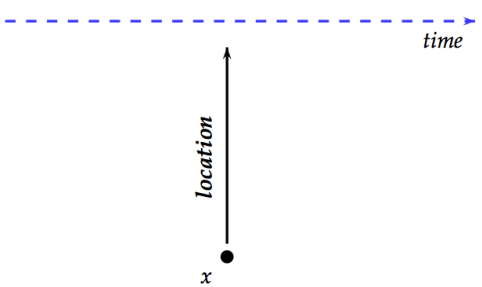

Transcendentism

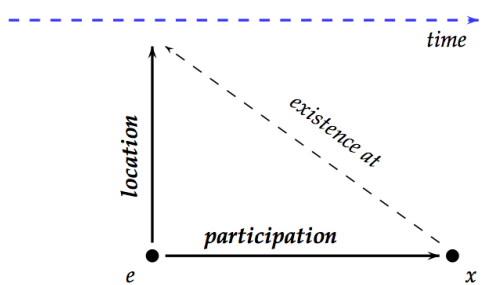

that are defined in terms of existence at a time. Most notably, persistence. According to both the immanentist and the transcendentist, for an object to persist means for it to exist at various times. The immanentist conflates existence at a time and weak location; hence, according to her, for an object to persist means for it to be weakly located at various times. More precisely, these times have to be taken to be instants, for an object that does not persist would anyway be weakly located at various times - the unique instant in which it comes into being and goes out of existence and all intervals overlapping it (Parsons, 2007). On the other hand, the transcendentist claims that for an object to exist at a time is for it to participate in events that are weakly located at that time; hence, according to her, for an object to persist means for it to participate in events that are weakly located at various times. Also in this case, these times have to be taken to be instants, for the same reason as before. Alternatively, a transcendentist can define the persistence of an object in terms of the temporal location of its history. In this case, we will say that an object persists just in case the temporal exact location of its history is temporally extended.

Since existence at a time has been introduced as a predicate that applies to objects only, also the aforementioned definitions of persistence can only apply to objects. However, objects are not the only entities that are said to persist 
through time. What should a transcendentist say about those other entities? Of course, it depends on the way in which such entities relate to time. If they are located in time, the original immanentist definitions will do. For example, I take events to be located in time. As a consequence, I would describe their persistence in immanentist terms: for them to persist is to be weakly located at various instants. Otherwise, if such entities are not located in time, it will be necessary to apply to them a strategy similar to the one applied to the case of objects.

Suppose you decide to go transcendentist. Does that mean that you are committing yourself to a particular theory of persistence? In other words, is transcendentism a form of endurantism or perdurantism? The good news is that transcendentism is an open option for everyone. Take perdurantism. A way of describing perdurantism goes as follows. Begin with an ontology in which no object persists through time. Simply, there is a series of instantaneous entities that are linked one to the other by a counterpart relation. Now, suppose you are also a mereological universalist. Those instantaneous entities will fuse together, and the fusions will be nothing but the perduring objects, and the instantaneous entities will be the temporal parts that the perdurantist knows and loves. Notice that this way of describing perdurantism does not make reference to temporal location. As a consequence, the perdurantist can save the spirit of her view in a transcendentist setting just as well as in an immanentist one.

Even if transcendentism can be conjoined with perdurantism, I think it does its best when conjoined with endurantism. Here I take the basic idea of endurantism to be that objects persist without having temporal parts. There is a clear sense in which this can be true in the transcendentist setting, i.e. that I - and not some proper part of me - am the participant of my life and all of its temporal parts. So, I take the transcendentist theory of persistence to be the 
claim that objects persist through time by being the participants of all temporal parts of their histories.

The advantages of this transcendentist theory of persistence are straightforward. Endurantists in particular should look at it with interest, because it allows them to make sense of objects' persistence through time without committing themselves to the problematic assumptions of other forms of endurantism. Recall that the extant versions of endurantism were committed to the possibility of multilocation or of extended simples. Given that transcendentism rejects the idea that objects are located at times altogether, it does not require - it actually denies - objects to be temporally multilocated, nor temporally extended.

The transcendentist theory of persistence may have further advantages, especially over the other forms of endurantism described before. For example, unlike multilocationism, it does not require time to be discrete. Recall that multilocationism has it that persisting objects are exactly located at instants of time. Hence, multilocationism is committed to instants of time. On the other hand, the transcendentist theory of persistence does not carry with it this committment. For another example, unlike simplism, the transcendentist theory of persistence allows objects to have spatial parts. Recal that simplism has it that objects are extended simples. If something is an extended simple, it lacks any proper parts, so spatial parts as well ${ }^{9}$. On the other hand, the transcendentist theory of persistence does not carry with it this committment.

\footnotetext{
${ }^{9}$ It is possible to use the core idea of simplism in order to define another theory of persistence that has no such committments. Yet, this theory still implies that objects cannot change their spatial parts (see author's manuscript).
} 


\section{Conclusion}

The focus of this work has been existence at a time - the relation between objects and time -. I have argued that such a relation should not be conflated with location and that analyzing it in terms of the events in which substances participate may reveal itself to be a semantically grounded and metaphysically fruitful choice. For reasons of space, I leave arguments that favour transcendentism as well as the discussion of some worries - such as a worry arising from special relativity as well as another according to which transcendentism makes events more fundamental than objects - for another forthcoming paper. However, what has been said so far seems to be enough to suggest that if one is going to be an endurantist, she should seriously consider to be a transcendentist endurantist.

\section{References}

Armstrong, D. M. (1968). A Materialist Theory of the Mind. Routledge.

Balashov, Y. (2010). Persistence and Spacetime. Oxford: Oxford University Press.

Barker, S. and Dowe, P. (2003). Paradoxes of Multilocation. Analysis, 63(2):106-114.

Barker, S. and Dowe, P. (2005). Endurance is Paradoxical. Analysis, 65(1):6974 .

Bennett, J. F. (1988). Events and their Names. Oxford: Clarendon Press.

Bittner, T. and Donnelly, M. (2006). A Classification of Spatio-temporal Entities Based on Their Location in Space-time. In International Workshop 
on Semantic-based Geographical Information Systems, pages 1626-1635. Dordrecht: Springer.

Braddon-Mitchell, D. (2003). Qualia and Analytical Conditionals. Journal of Philosophy, 100(3):111-135.

Braddon-Mitchell, D. and Miller, K. (2006). The Physics of Extended Simples. Analysis, 66(291):222-226.

Casati, R. and Varzi, A. C. (1996). The Structure of Spatial Localization. Philosophical Studies, 82(2):205-239.

Casati, R. and Varzi, A. C. (1999). Parts and Places: the Structures of Spatial Representations. Cambridge, Mass.: MIT Press.

Churchland, P. M. (1981). Eliminative Materialism and the Propositional Attitudes. Journal of Philosophy, 78:67-90.

Churchland, P. M. (1984). Matter and Consciousness. MIT Press.

Churchland, P. S. (1986). Neurophilosophy: Toward A Unified Science of the Mind-Brain. MIT Press.

Correia, F. (2005). Existential Dependence and Cognate Notions. Munich: Philosophia Verlag.

Correia, F. and Schnieder, B. (2012). Grounding: An Opinionated Introduction. In Correia, F. and Schnieder, B., editors, Metaphysical Grounding: Understanding the Structure of Reality, pages 1-36. Cambridge University Press.

Dasgupta, S. (2014). The Possibility of Physicalism. Journal of Philosophy, 111(9/10):557-592.

deRosset, L. (2013). Grounding Explanations. Philosophers' Imprint, 13(7). 
Dretske, F. (1967). Can Events Move? Mind, 76(304):479-492.

Effingham, N. (2015). The Location of Properties. Nô̂s, 49(2).

Effingham, N. and Robson, J. (2007). A Mereological Challenge to Endurantism. Australasian Journal of Philosophy, 85(4):633-640.

Ehring, D. (2002). Spatial Relations between Universals. Australasian Journal of Philosophy, 80(1):17-23.

Fine, K. (2006). In Defence of Three-Dimensionalism. Journal of Philosophy, 103:699-714.

Fine, K. (2012). Guide to Ground. In Correia, F. and Schnieder, B., editors, Metaphysical Grounding, pages 37-80. Cambridge: Cambridge University Press.

Galton, A. (2006). Processes as Continuants. In Proceedings of the International Workshop on Temporal Representation and Reasoning, pages 187-187.

Geach, P. T. (1969). God and the Soul. London: Routledge.

Gilmore, C. (2003). In Defence of Spatially Related Universals. Australasian Journal of Philosophy, 81(3):420-428.

Gilmore, C. (2004). Material Objects: Metaphysical Issues. PhD thesis.

Gilmore, C. (2006). Where in the Relativistic World Are We? Philosophical Perspectives, 20(1):199-236.

Gilmore, C. (2007). Time-travel, Coinciding Objects, and Persistence. In Bennett, K. and Zimmerman, D. W., editors, Oxford studies in Metaphysics, volume 3, pages 177-198. Oxford: Clarendon Press.

Gilmore, C. (2008). Persistence and Location in Relativistic Spacetime. Philosophy Compass, 3(6):1224-1254. 
Hawley, K. (2001). How Things Persist. Oxford: Oxford University Press.

Hofweber, T. and Velleman, J. D. (2011). How to Endure. The Philosophical Quarterly, 61(242):37-57.

Jackson, F. (2003). Mind and Illusion. In O'Hear, A., editor, Royal Institute of Philosophy Supplement, volume 53, pages 421-442. Cambridge University Press.

Kim, J. (1976). Events as Property Exemplifications. In Brand, M. and Walton, D., editors, Action theory, pages 159-177. Dordrecht: D. Reidel.

King, J. (1998). What is a Philosophical Analysis? Philosophical Studies, 90(2):155-179.

Kleinschmidt, S. (2011). Multilocation and Mereology. Philosophical Perspectives, 25(1):253-276.

Lewis, D. (1970). An Argument for the Identity Theory. Journal of Philosophy, 63(2):17-25.

Lewis, D. K. (1986). On the Plurality of Worlds. Oxford: Blackwell.

Lombard, L. B. (1986). Events: A Metaphysical Study. London: Routledge.

Lowe, E. J. (1998). The Possibility of Metaphysics: Substance, Identity, and Time: Substance, Identity, and Time. Oxford: Oxford University Press.

Mellor, D. H. (1998). Real Time II. London: Routledge.

Parsons, J. (2000). Must a Four-dimensionalist Believe in Temporal Parts? the Monist, 83(3):399-418.

Parsons, J. (2007). Theories of Location. In Bennett, K. and Zimmerman, D. W., editors, Oxford Studies in Metaphysics, volume 3, pages 201-32. Oxford: Clarendon Press. 
Rorty, R. (1965). Mind-Body Identity, Privacy, and Categories. Review of Metaphysics, 19:24-54.

Rosen, G. (2010). Metaphysical Dependence: Grounding and Reduction. In Hale, B. and Hoffmann, A., editors, Modality: Metaphysics, Logic, and Epistemology, pages 109-36. New York: Oxford University Press.

Russell, B. (1903). The Principles of Mathematics. Cambridge: Cambridge University Press.

Sattig, T. (2006). The Language and Reality of Time. Oxford: Clarendon Press.

Schaffer, J. (2009). On What Grounds What. In Chalmers, D., M. D. and Wasserman, R., editors, Metametaphysics, pages 347-383. Oxford: Oxford University Press.

Schaffer, J. (2012). Grounding, Transitivity, and Contrastivity. In Correia, F. and Schnieder, B., editors, Metaphysical Grounding. Cambridge: Cambridge University Press.

Schnieder, B. (2011). A Logic for "Because". Review of Symbolic Logic, 4:445465.

Sider, T. (1997). Four-dimensionalism. The Philosophical Review, 106(2):197231.

Sider, T. (2001). Four-dimensionalism: An Ontology of Persistence and Time. Oxford: Oxford University Press.

Simons, P. (2014). Where It's At: Modes of Occupation and Kinds of Occupant. In Kleinschmidt, S., editor, Mereology and Location. Oxford: Oxford University Press.

Stich, S. (1983). From Folk Psychology to Cognitive Science. MIT Press. 
Stout, R. (1997). Processes. Philosophy, 72:19-27.

van Inwagen, P. (1990). Four-Dimensional Objects. Noûs, 24(2):245-255.

Varzi, A. C. (2003). Naming the Stages. dialectica, 57(4):387-412. 